\title{
DIFFERENTIABILITY VIA DIRECTIONAL DERIVATIVES
}

\author{
KA-SING LAU AND CLIFFORD E. WEIL
}

\begin{abstract}
Let $F$ be a continuous function from an open subset $D$ of a separable Banach space $X$ into a Banach space $Y$. We show that if there is a dense $G_{\delta}$ subset $A$ of $D$ and a $G_{\delta}$ subset $H$ of $X$ whose closure has nonempty interior, such that for each $a \in A$ and each $x \in H$ the directional derivative $D_{x} F(a)$ of $F$ at $a$ in the direction $x$ exists, then $F$ is Gâteaux differentiable on a dense $G_{\delta}$ subset of $D$. If $X$ is replaced by $R^{n}$, then we need only assume that the $n$ first order partial derivatives exist at each $a \in A$ to conclude that $F$ is Frechet differentiable on a dense, $G_{\delta}$ subset of $D$.
\end{abstract}

1. Introduction. It is known that if $F$ is a real valued function on an open subset $D$ in $R^{n}$ whose first order partial derivatives exist and are continuous, then $F$ is differentiable on $D$. Stepanoff [7] showed that there exists a continuous function on $R^{2}$ such that the partial derivatives exist almost everywhere but the function is nowhere differentiable. In contrast to this result, we prove in this note that the existence of partial derivatives on a dense $G_{\delta}$ subset will imply that the function is differentiable on a dense $G_{\delta}$ subset.

Throughout, we assume the scalar field is $R$. Let $X$ and $Y$ be locally convex spaces and let $F$ be a function from an open subset $D$ of $X$ into $Y$. We say that $F$ is Gâteaux differentiable at $a \in D$ if there exists a continuous linear operator $D F(a): X \rightarrow Y$ such that for any $x \in X$,

$$
D F(a)(x)=\lim _{t \rightarrow 0} t^{-1}(F(a+t x)-F(a))
$$

(the limit depends on $x$ ). We call $D F(a)$ the Gâteaux differential of $F$ at $a$. When the above limit exists it is called the directional derivative of $F$ at $a$ in the direction $x$ and is denoted by $D_{x} F(a)$. The reader may refer to [9] for a detailed discussion of the Gâteaux derivative and its relations with other derivatives. Our main theorem is the following: Suppose $F$ is a continuous function from an open subset $D$ of a separable Banach space $X$ into a Banach space $Y$ and suppose there exists a dense $G_{\delta}$ subset $A$ of $D$ and a $G_{\delta}$ subset $H$ of $X$ whose closure has nonempty interior such that for $a \in A$, the directional derivative $D_{x} F(a)$ exists for each $x \in H$. Then $F$ is Gâteaux differentiable on a dense $G_{\delta}$ of $D$.

In $\S 2$, we give some lemmas. In $\S 3$, we prove the main theorem as well as some other related results.

Received by the editors April 16, 1976.

AMS (MOS) subject classifications (1970). Primary 26A54; Secondary 46B99.

Key words and phrases. Baire space, dense $G_{\delta}$, directional, Gâteaux, and Frechet derivatives. 
2. Some lemmas. A (Hausdorff) topological space $X$ is called a Baire space if the intersection of any sequence of open dense subsets in $X$ is dense in $X$. It may be shown that dense $G_{\delta}$ subsets and open subsets of Baire spaces and, of course, complete metric spaces are Baire spaces.

We omit the proof of the following obvious lemma.

LEMMA 2.1. Let $G_{n}$ be a sequence of open subsets in a Baire space $X$ with $\cup_{n=1}^{\infty} G_{n}$ dense in $X$. Let $A$ be a subset of $X$ such that for each $n, A \cap G_{n}$ contains a dense $G_{\delta}$ in $G_{n}$. Then $A$ contains a dense $G_{\delta}$ in $X$.

Let $X$ and $Y$ be two sets and let $A$ be a subset of $X \times Y$. For each $x \in X$, we use $A_{x}$ to denote the $x$-section of $A$; i.e. $A_{x}=\{y \in Y:(x, y) \in A\}$.

LEMMA 2.2. Let $X$ and $Y$ be Baire spaces with $Y$ second countable, and let $A$ be a dense $G_{\delta}$ subset of $X \times Y$. Then $\left\{x \in X: A_{x}\right.$ is a dense $G_{\delta}$ subset of $\left.Y\right\}$ contains a dense $G_{\delta}$ subset of $X$.

Proof. Assume first that $A$ is an open dense subset of $X \times Y$. Let $A=\cup_{\alpha}\left(U_{\alpha} \times V_{\alpha}\right)$, where $U_{\alpha}$ and $V_{\alpha}$ are open subsets of $X$ and $Y$, respectively, let $\left\{W_{n}\right\}$ be a countable base for the topology on $Y$, and for each $n$ let $B_{n}=\cup_{\alpha}\left\{U_{\alpha}: V_{\alpha} \cap W_{n} \neq \varnothing\right\}$. It is clear that $B_{n}$ is open and easy to show that $B_{n}$ is dense in $X$. Since $X$ is a Baire space, $B=\bigcap_{n=1}^{\infty} B_{n}$ is a dense $G_{\delta}$ subset of $X$. Let $x \in B$. For each $n$ there exists an $\alpha$ such that $x \in U_{\alpha}$ and $V_{\alpha} \cap W_{n} \neq \varnothing$. It follows that $A_{x}$ is dense in $Y$. Noting that $A_{x}$ is open completes the proof in the case where $A$ is open.

Let $A=\bigcap_{n=1}^{\infty} A_{n}$ where each $A_{n}$ is an open dense subset of $X \times Y$. For each $n$ there is a dense $G_{\delta}$ subset $B_{n}$ of $X$ such that $B_{n} \subseteq\left\{x \in X:\left(A_{n}\right)_{x}\right.$ is a dense $G_{\delta}$ subset of $Y$ \}. Let $B=\bigcap_{n=1}^{\infty} B_{n}$. Since $X$ is a Baire space, $B$ is a dense $G_{\delta}$ subset of $X$. Let $x \in B$. Then for each $n\left(A_{n}\right)_{x}$ is a dense $G_{\delta}$ subset of $Y$. Since $Y$ is a Baire space, $\cap_{n=1}^{\infty}\left(A_{n}\right)_{x}$ is a dense $G_{\delta}$ subset of $Y$. But $\bigcap_{n=1}^{\infty}\left(A_{n}\right)_{x}=A_{x}$, which completes the proof.

LEMMA 2.3. Let $X$ be a Baire space, let $Y$ be a metric space, and let $F$ : $X \times\{t: 0<|t|<r\} \rightarrow Y$, where $r$ is a fixed positive number. Suppose that for each $0<|t|<r, F(x, t)$ is continuous in $x$, and that there is a dense $G_{\delta}$ set $A$ in $X$ such that $\lim _{t \rightarrow 0} F(a, i)=F_{0}(a)$ exists for all $a \in A$. Then there is $a$ dense $G_{\delta}$ set $E$ in $X$ such that for each $a \in E$,

$$
\lim _{(x, t) \rightarrow(a, 0), t \neq 0} F(x, t)=F_{0}(a) \text {. }
$$

Proof. $F_{0}$ is a function of Baire class 1. Let $E_{1}=\left\{a \in A: F_{0}\right.$ is continuous at $a$ \}. Then $E_{1}$ is a dense $G_{\delta}$ set in $A$ and, hence, in $X$ [3, Theorem 2]. Let $M$ and $N$ be positive integers, and let

$$
\begin{aligned}
& A_{M N}=\left\{x \in X: \text { if } 0<|t|<N^{-1}\right. \text { and } \\
& \left.\qquad 0<|s|<N^{-1} \text {, then } \rho(F(x, t), F(x, s)) \leqslant M^{-1}\right\} .
\end{aligned}
$$

Then $A_{M N}$ is a closed set and, for each $M, \cup_{N=1}^{\infty} A_{M N} \supseteq A$. So since $X$ is a Baire space, $G_{M}=\cup_{N=1}^{\infty} A_{M N}^{0}$ is an open dense subset of $X$. Let $E_{2}=$ 
$\cap_{M=1}^{\infty} G_{M}$. Since $X$ is a Baire space, $E_{2}$ is a dense $G_{\delta}$ set in $X$ as is $E=E_{1} \cap E_{2}$.

Let $a \in E$ and let $\varepsilon>0$. Since $F_{0}$ is continuous at $a$ there is a neighborhood $U_{1}$ of $a$ such that if $y \in U_{1} \cap A$, then $\rho\left(F_{0}(y), F_{0}(a)\right)<\varepsilon / 2$. There is a positive integer $M$ such that $M^{-1}<\varepsilon / 2$. Since $a \in E_{2}, a \in G_{M}$. Thus there is an $N$ such that $a \in A_{M N}^{0}$. Let $U=U_{1} \cap A_{M N}^{0}$. Let $x \in U$ and $0<|t|<$ $N^{-1}$. Then for each $y \in U \cap A$,

$$
\begin{aligned}
\rho\left(F(x, t), F_{0}(a)\right) \leqslant & \rho(F(x, t), F(y, t)) \\
& +\rho\left(F(y, t), F_{0}(y)\right)+\rho\left(F_{0}(y), F_{0}(a)\right) .
\end{aligned}
$$

Since $U \cap A$ is dense in $U$ and since $F(y, t)$ is continuous as a function of $y$,

$$
\lim _{y \rightarrow x} \rho(F(x, t), F(y, t))=0 .
$$

Since $y \in U \cap A$, and since $0<|t|<N^{-1}$,

$$
\rho\left(F(y, t), F_{0}(y)\right)=\lim _{s \rightarrow 0} \rho(F(y, t), F(y, s)) \leqslant M^{-1} .
$$

Thus

$$
\limsup _{y \rightarrow x} \rho\left(F(y, t), F_{0}(y)\right) \leqslant M^{-1}<\varepsilon / 2 .
$$

Consequently, $\rho\left(F(x, t), F_{0}(a)\right)<\varepsilon$.

Lemma 2.4. Let $X$ be a Banach space and let $A$ be a dense $G_{\delta}$ in an open subset $U$ of $X$. Then the linear hull of $A$ equals $X$.

Proof. Suppose $\operatorname{lin} A \neq X$. Then there exists an $x$ in $X \backslash \operatorname{lin} A$ so that $U \cap(U+x) \neq \varnothing$. It is easy to see that both $A \cap U \cap(U+x)$ and $(A+$ $x) \cap U \cap(U+x)$ contain dense $G_{\delta}$ subsets of $U \cap(U+x)$ and are disjoint, which is a contradiction.

\section{The theorems.}

THEOREM 3.1. Let $X$ and $Y$ be separable Banach spaces. Let $D$ be an open subset of $X$, and let $F: D \rightarrow Y$ be continuous. Suppose there is a dense $G_{\delta}$ subset $A$ of $D$ such that for each $a \in A$, the directional derivative $D_{x} F(a)$ exists for each $x \in H$ where $H$ is a $G_{\delta}$ subset of $X$ which is dense in a nonempty open subset $U$ of $X$. Then $F$ is Gâteaux differentiable on a dense $G_{\delta}$ subset of $D$.

Proof. For each positive integer $k$ let

$$
\begin{aligned}
A_{k}=\left\{(a, x) \in D \times U: \| t^{-1}(F(a+t x)-\right. & F(a)) \| \leqslant k \\
& \text { for } \left.0<|t|<k^{-1}\right\} .
\end{aligned}
$$

Since $F$ is continuous, $A_{k}$ is closed, and since $D_{x} F(a)$ exists for each $a \in A$ and $x \in H, A \times H \subseteq \cup \cup_{k=1}^{\infty} A_{k}$. Thus $\cup_{k=1}^{\infty} A_{k}$ is dense in $D \times U$, which is an open subset of the complete metric space $X \times X$ and, consequently, a Baire space. It follows that $\cup_{k=1}^{\infty} A_{k}^{0}$ is dense in $D \times U$. Note that each $A_{k}^{0}$ is the countable union of $D_{k j} \times U_{k j}$, where $D_{k j}$ is open in $D$ and $U_{k j}$ is open in $U$. By Lemma 2.1 it suffices to prove that for each $j D_{k j}$ contains a suitable 
dense $G_{\delta}$ set. For notational ease $D_{k j}$ and $U_{k j}$ will be denoted simply by $D_{k}$ and $U_{k}$, respectively.

Define $\tilde{F}: D_{k} \times U_{k} \times\left\{t: 0<|t|<k^{-1}\right\} \rightarrow Y$ by

$$
\tilde{F}(a, x, t)=t^{-1}(F(a+t x)-F(a)) \text {. }
$$

For each $(a, x) \in\left(A \cap D_{k}\right) \times\left(H \cap U_{k}\right), \lim _{t \rightarrow 0} \tilde{F}(a, x, t)=D_{x} F(a) . A$ $\cap D_{k}$ and $H \cap U_{k}$ are dense $G_{\delta}$ subsets of $D_{k}$ and $U_{k}$, respectively. Hence $\left(A \cap D_{k}\right) \times\left(H \cap U_{k}\right)$ is a dense $G_{\delta}$ subset of $D_{k} \times U_{k}$ and, as was pointed out in the above paragraph, $D_{k} \times U_{k}$ is a Baire space. So by Lemma 2.3 there exists a dense $G_{\delta}$ subset $E$ of $\left(A \cap D_{k}\right) \times\left(H \cap U_{k}\right)$ such that for each $(a, x) \in E$,

$$
\lim _{\left(a^{\prime}, x^{\prime}, t\right) \rightarrow(a, x, 0), t \neq 0} \tilde{F}\left(a^{\prime}, x^{\prime}, t\right)=D_{x} F(a) .
$$

By Lemma 2.2 there is a dense $G_{\delta}$ subset $B$ of $A \cap D_{k}$, which is therefore a dense $G_{\delta}$ subset of $D_{k}$, such that for each $a \in B, E_{a}$ is a dense $G_{\delta}$ subset of $H \cap U_{k}$ and, consequently, a dense $G_{\delta}$ subset of $U_{k}$.

Let $a \in B, x \in E_{a}$ and $y \in X$ such that $D_{y} F(a)$ exists. Then

$$
\begin{aligned}
\lim _{t \rightarrow 0} t^{-1}( & F(a+t(x+y))-F(a)) \\
= & \lim _{t \rightarrow 0} t^{-1}(F(a+t x+t y)-F(a+t y)) \\
& +\lim _{t \rightarrow 0} t^{-1}(F(a+t y)-F(a)) \\
= & \lim _{(a+t y, x, t) \rightarrow(a, x, 0)} \tilde{F}(a+t y, x, t)+D_{y} F(a) \\
= & D_{x} F(a)+D_{y} F(a) .
\end{aligned}
$$

So $D_{x+y} F(a)$ exists and $D_{x+y} F(a)=D_{x} F(a)+D_{y} F(a)$. Using the obvious fact that if $D_{x} F(a)$ exists and if $\alpha \in R$, then $D_{\alpha x} F(a)$ exists and $D_{\alpha x} F(a)=$ $\alpha D_{x} F(a)$, it is an easy matter to establish that

$\left\{x \in X: D_{x} F(a)\right.$ exists and for each $y$ such that $D_{y} F(a)$ exists, $D_{x+y} F(a)$ exists and $D_{x+y} F(a)=D_{x} F(a)+$ $\left.D_{y} F(a)\right\}$

is a subspace of $X$. Since it contains $E_{a}$ Lemma 2.4 implies that it is $X$. That is, for all $x \in X, D_{x} F(a)$ exists and for each $y \in X, D_{x+y} F(a)=D_{x} F(a)+$ $D_{y} F(a)$. Thus the operator $(D F(a))(x)=D_{x} F(a)$ is linear. Since $D_{k} \times U_{k} \subseteq$ $A_{k}$, for all $x \in U_{k},\left\|D_{x} F(a)\right\| \leqslant k$; that is, the linear operator is bounded on a nonempty open set $U_{k}$ and, hence, is bounded.

If $X=R^{n}$, we let $\left\{e_{1}, \ldots, e_{n}\right\}$ be the natural basis, and let $D_{i} F$ denote the partial derivative of $F$ in the $i$ th coordinate.

TheOREM 3.2. Let $D \subseteq R^{n}$ be open, let $Y$ be a separable Banach space, and let $F: D \rightarrow Y$ be continuous. Suppose there is a dense $G_{\delta}$ subset $A$ of $D$ such that for each $a \in A$ and each $i=1, \ldots, n, D_{i} F(a)$ exists. Then $F$ is (Frechet) differentiable on a dense $G_{\delta}$ subset of $D$. 
Proof. For $r>0$ let $D_{r}=\left\{x \in D: \operatorname{dist}\left(x, R^{n} \backslash D\right)>r\right\}$. By Lemma 2.1 we need only prove the theorem on $D_{r}$ for each $r>0$. For each $i=1, \ldots, n$ define $\tilde{F}_{i}: D_{r} \times\{t: 0<|t|<r\}$ by

$$
\tilde{F}_{i}(a, t)=t^{-1}\left(F\left(a+t e_{i}\right)-F(a)\right) .
$$

Then for each $i=1, \ldots, n$ and each $a \in A \cap D_{r}, \lim _{t \rightarrow 0} \tilde{F}_{i}(a, t)=D_{i} F(a)$.

By Lemma 2.3 for each $i=1, \ldots, n$ there is a dense $G_{\delta}$ subset $A_{i}$ of $D_{r}$ such that for each $a \in A_{i}$,

$$
\lim _{\left(a^{\prime}, t\right) \rightarrow(a, 0), t \neq 0} \tilde{F}\left(a^{\prime}, t\right)=D_{i} F(a) .
$$

Let $E=\cap_{i=2}^{n} A_{i}$. Then $E$ is a dense $G_{r \delta}$ ubset of $D_{r}$.

Let $a \in E$. For each $x \in R^{n} \backslash\{0\}$ write $x=\sum_{j=1}^{n} \lambda_{j} e_{j}$, and for each $i=$ $2, \ldots, n+1$, let $a_{i}=a+\sum_{j=1}^{i-1} \lambda_{j} e_{j}$. Then

$$
\begin{aligned}
\lim _{x \rightarrow 0}|x|^{-1} \mid & \left(F(a+x)-F(a)-\sum_{i=1}^{n} \lambda_{i} D_{i} F(a)\right) \mid \\
\leqslant & \sum_{i=2}^{n} \lim _{x \rightarrow 0}|x|^{-1}\left|\left(F\left(a_{i+1}\right)-F\left(a_{i}\right)-\lambda_{i} D_{i} F(a)\right)\right| \\
& \quad+\lim _{x \rightarrow 0}|x|^{-1}\left|\left(F\left(a+\lambda_{1} e_{1}\right)-F(a)-\lambda_{1} D_{1} F(a)\right)\right| \\
\leqslant & \sum_{i=2}^{n} \lim _{\left(a_{i}, \lambda_{i}\right) \rightarrow(a, 0)}\left|\tilde{F}\left(a_{i}, \lambda_{i}\right)-D_{i} F(a)\right| \\
& +\lim _{\lambda_{1} \rightarrow 0}\left|\lambda_{1}^{-1}\left(F\left(a+\lambda_{1} e_{1}\right)-F(a)\right)-D_{1} F(a)\right| \\
= & 0 .
\end{aligned}
$$

Suppose $F$ is a continuous function from an open subset $D$ of $R^{n}$ into a Banach space $Y$ and suppose that $D_{i} F, i=1, \ldots, n$, exists on a dense $G_{\delta}$ subset $A$ in $D$. Then $F$ satisfies the local Lipschitz condition on an open, dense subset in $D$. Indeed, if for each positive integer $k$,

$$
A_{k}=\left\{a \in D:\left\|t^{-1}\left(F\left(a+t e_{i}\right)-F(a)\right)\right\|<k, \quad|t| \leqslant k^{-1}, i=1, \ldots, n\right\},
$$

then each $A_{k}$ is closed and $\cup_{k=1}^{\infty} A_{k}$ contains the set $A$. Hence the set $U=\bigcup_{k=1}^{\infty} A_{k}^{0}$ is an open dense set in $D$ and $F$ satisfies the local Lipschitz condition at each point $a \in U$.

THEOREM 3.3. Let $F$ be a continuous function from an open subset $D \subseteq R^{n}$ into $R^{m}$. Suppose there exists a dense $G_{\delta}$ subset $A \subseteq D$ such that $D_{i} F(a)$, $i=1, \ldots, n$, exist for each $a \in D$. Then $F$ is differentiable on $a$ dense, measurable subset in $D$ with positive (Lebesgue) measure.

Proof. It follows from the above remark that $F$ satisfies the local Lipschitz condition on an open, dense subset $U$ in $D$. By a theorem of Rademacher ([6], cf. also [2, p. 218]), $F$ is differentiable a.e. on $U$ and, hence, $F$ is 
differentiable on a dense set with positive measure.

The theorem of Rademacher on the differentiability of Lipschitz mappings has been generalized by Mankiewicz [4], [5] and Christensen [1] into some classes of infinite dimensional Banach spaces. However, we remark that a Lipschitz function is not necessarily differentiable on any dense $G_{\delta}$ subset; an example was given by Zahorski in [8].

In the rest of this section, we will consider a map $F$ from an open subset $D$ of a separable Banach space $X$ into a dual Banach space $Y^{*}$ with the weak* topology. The definition of Gâteaux differentiable of $F$ at $a$ becomes: there exists a bounded linear operator $D F(a)$ from $X$ into $Y^{*}$ such that

$$
\langle D F(a) x, y\rangle=\lim _{t \rightarrow 0} t^{-1}\langle F(a+t x)-F(a), y\rangle \text { for all } x \in X, y \in Y .
$$

For convenience, we will call this the $w^{*}$-Gâteaux differential of $F$ at $a$. We also call a function $F: D \rightarrow Y^{*}$ demicontinuous if $F$ is continuous with respect to the weak* topology in $Y^{*}$.

Theorem 3.4. Let $X$ and $Y$ be separable Banach spaces. Let $D$ be an open subset of $X$ and let $F$ be a demicontinuous function from $D$ into $Y^{*}$. Suppose there exists a dense $G_{\delta}$ subset $A$ of $D$ such that for each $a \in A, D_{x} F(a)$ exists for each direction $x \in X$. Then $F$ is $w^{*}$-Gateaux differentiable on a dense $G_{\delta}$ in D.

Proof. For each $a \in A, x \in X, 0<\delta$ sufficiently small and $y \in Y$, $t^{-1}\langle F(a+t x)-F(a), y\rangle$ is a continuous function for $|t| \leqslant \delta$. Consequently, $\left\{t^{-1}\langle F(a+t x)-F(a), y\rangle: 0<|t|<\delta\right\}$ is a bounded set. By the uniformly boundedness principle, the set $\left\{\left\|t^{-1}(F(a+t x)-F(a))\right\|: 0<|t|\right.$ $<\delta\}$ is also a bounded set. Let

$$
A_{k}=\left\{(a, x) \in D \times X:\left\|t^{-1}(F(a+t x)-F(a))\right\| \leqslant k \text { for all }|t|<k^{-1}\right\} .
$$

By the demicontinuity and the lower semicontinuity of the norm, each $A_{k}$ is closed in $D \times X$. Since $\cup_{k=1}^{\infty} A_{k} \supseteq A \times X$, the set $\cup_{k=1}^{\infty} A_{k}^{0}$ is an open dense subset in $D \times X$.

It follows from the same argument as Theorem 3.1 that it suffices to prove the theorem on any open subset $D_{k}$ where $D_{k} \times U_{k} \subseteq A_{k}^{0}$. The rest of the proof is also the same as Theorem 3.1; the only changes are: (i) when applying Lemma 2.3 to $\tilde{F}$, we have to observe that $Y$ is separable, the image of $\tilde{F}$, which is contained in a bounded set in $Y^{*}$, is $w^{*}$-metrizable; (ii) in the proof of $D_{x+y} F(a)=D_{x} F(a)+D_{y} F(a)$ we change

$$
\lim _{t \rightarrow 0} t^{-1}(F(a+t(x+y))-F(a))=\text { etc. }
$$

into

$$
\lim _{t \rightarrow 0}\left\langle t^{-1}(F(a+t(x+y))-F(a)), y\right\rangle=\text { etc. }
$$

for each $y \in Y$. 


\section{REFERENCES}

1. J. Christensen, Topology and Borel structure, North-Holland, Amsterdam; American Elsevier, New York, 1973.

2. H. Federer, Geometric measure theory, Springer-Verlag, New York, 1969.

3. M. Fort, Jr., Category theorems, Fund. Math. 42 (1955), 276-288.

4. P. Mankiewicz, On the differentiability of Lipschitz mappings in Fréchet spaces, Studia Math. 45 (1973), 15-29.

5. On topological, Lipschitz and uniform classification of LF-spaces, Studia Math. 52 (1974), 107-142.

6. H. Rademacher, Über partielle und totale Differenzierbarkeit, Math. Ann. 79 (1919), 340-359.

7. W. Stepanoff, Sur le conditions de l'existence de la differentielle totale, Rec. Math. Soc. Math. Moscow 32 (1925), 511-526.

8. Z. Zahorski, Sur l'ensemble des points de non dérivabilité d'une fonction continue, Bull. Soc. Math. France 74 (1946), 147-178.

9. S. Yamamuro, Differential calculus in topological linear spaces, Lecture Notes in Math., no. 374, Springer-Verlag, Berlin and New York, 1974.

Department of Mathematics, University of Pittsburgh, Pittsburgh, Pennsylvania 15260

Department of Mathematics, Michigan State University, East Lansing, Michigan 48823 